\title{
Composition Determination of Rubber Blends by Applying Differential Scanning Calorimetry and SPA-PLS Treatment
}

\author{
Jordi-Roger Riba ${ }^{a}{ }^{\infty}$, Marcela Ángela Mansilla ${ }^{b}$, Trini Canals $^{c}$, Rosa Cantero ${ }^{c}$ \\ ${ }^{a}$ Electrical Engineering Department, Universitat Politècnica de Catalunya, Rambla St. Nebridi 22, \\ 08222 Terrassa, Spain \\ ${ }^{b}$ Instituto Nacional de Tecnología Industrial - INTI, Consejo Nacional de Investigaciones Cientificas y \\ Técnicas - CONICET, Centro de Caucho, Av. General Paz 5445, B1650WAB San Martín, Argentina \\ ${ }^{c}$ Escola Politècnica Superior, Universitat de Lleida, Pla de la Massa 8, 08700 Igualada, Spain
}

Received: June 14, 2018; Revised: October 01, 2018; Accepted: November 08, 2018

\begin{abstract}
This paper proposes an innovative approach to determine the composition of natural rubber (NR) and styrene butadiene rubber (SBR) blends from the analysis of the data provided by the differential scanning calorimetry (DSC) technique. DSC registers are post-processed, based on a multivariate chemometric approach, consisting in the application of the successive projections algorithm (SPA) as variable selection algorithm and a further application of the partial least squares (PLS) regression method and the leave-one-out cross-validation algorithm. Results attained by applying this approach are compared with those obtained from mid-infrared spectroscopy since this last method has been widely applied during the last decades. Experimental results summarized in the paper prove that this fast and cost-effective approach is useful to determine the composition of unknown incoming NR/SBR blends.
\end{abstract}

Keywords: Differential Scanning Calorimetry, Partial least squares, Natural rubber, Composition.

\section{Introduction}

Polymer materials have achieved a vital position in all branches of science and technology. Natural rubber (NR) is a natural homopolymer obtained from latex, a milky emulsion obtained from some plant types. NR is being used in many applications including balloons, surgical gloves, tires, or suspension element in bridges and buildings, due to its high elasticity, low-temperature flexibility, high fatigue and tearing resistance, low heat buildup and low production cost among others. Therefore, NR is among the most important polymers because of its widespread use and importance in many industrial areas ${ }^{1}$. Another advantage is that NR is obtained from a renewable resource, as opposed to the synthetic rubbers.

Styrene-butadiene rubber (SBR) is a general purpose synthetic copolymer obtained from the polymerization of styrene and butadiene. SBR designs a variety of rubbers which differentiate because of the different proportions of styrene and butadiene, the additives applied for processing and the polymerization type. SBR is applied in several engineering and industrial applications due to the appealing features, including high filler-loading capacity, good flex resistance, crack-initiation resistance, and abrasion resistance. The styrene/butadiene fraction ratio has a deep impact on the glass transition temperature, $T_{g}$, and thus in the physical properties of the material. The $T_{g}$ depends on the microstructure of the

*e-mail: riba@ee.upc.edu polymer and it is associated to the brittleness of the SBR compound at low temperatures ${ }^{2}$.

Natural rubber and styrene-butadiene rubber, in their pure states, have poor mechanical properties and virtually no technological application. The vulcanization of the elastomer produces profound changes at the molecular level, because of the formation of bridges or cross-links between polymer chains, thus forming a crosslinked three-dimensional structure. This modification produces important changes in the material since the physical properties are transformed from soft, weak and with high viscosity to exhibit high elasticity, less grip, insolubility and resistant to the premature aging caused by light, heat or other factors.

NR and SBR materials technology needs extensive compounding. A representative recipe includes the elastomeric polymer, plasticizers, fillers, antidegradants, flow agents, vulcanizing agents, accelerator and activators among others. The properties of the final product highly rely on the particular compound formulation and processing method applied ${ }^{3}$.

Since developing new polymeric materials is expensive, polymers are often blended to satisfy industry's need for high performance materials ${ }^{4,5}$. Natural rubber can be blended with SBR for obtaining enhanced oxidative stability performance. The mechanical and physical properties of the blended material are sensitive to small changes in the NR/SBR ratio ${ }^{6}$. Therefore, there is a need for developing direct, fast and accurate methods to evaluate the composition of such blends when the composition is not known.

DSC (differential scanning calorimetry) is a technique with more than 50 years of history ${ }^{7}$ which has been 
commonly applied to characterize different polymer blends ${ }^{8,9}$ of commercial importance. DSC has been also applied to determine the rubber composition of waste tires ${ }^{10}$, the curing temperature of nitrile rubbers ${ }^{11}$ or to control the curing of epoxy resins ${ }^{12}$, among others. The DSC patterns characteristic of each elastomer are kept in the blend, thus allowing to identify the polymers in the blend ${ }^{5}$. DSC allows analyzing the melting, miscibility and crystallization behavior of polymer blends ${ }^{13}$. Other techniques such as thermogravimetric analysis (TGA) can also be used to determine the composition of the polymers blends and their properties ${ }^{5}$, to measure the stability against temperature ${ }^{14}$ or the thermal degradation because of the simplicity of the weight loss technique. The Fourier transform infrared spectroscopy (FTIR) has also been applied to determine the composition of polymer blends ${ }^{15-17}$ since it allows to find structural changes and analyze specific bonds and interactions ${ }^{18}$. The thermal stability of the rubber samples has been investigated by applying TGA in combination with FTIR ${ }^{19}$.

Because of their relevance, this paper analyzes NR/SBR blends with variable blend ratios, which were vulcanized with a recipe commonly used in the industry. The compounds were analyzed by differential scanning calorimetry (DSC), and Fourier transform infrared (FTIR) spectroscopy as a comparison method. The DSC registers and the FTIR spectra of the studied sample blends were analyzed by multivariate chemometric methods in order to establish a mathematical correlation between those registers and the composition of the blend. The aim of the paper is to extract chemically relevant information of the samples from the data recorded to generate a mathematical calibration model to determine the composition of unknown incoming NR/SBR blends. This is a new approach since it is not common to apply this method in the analysis of rubber compounds. The choice of this NR/SBR blends is adequate because they are well characterized by different techniques usually applied in polymer science ${ }^{20-22}$.

The successive projections algorithm (SPA) ${ }^{23,24}$ jointly with the partial least squares (PLS) regression and the leaveone-out cross-validation (LOOCV) method have been applied as multivariate methods to generate the regression model that relates the DSC registers and the FTIR spectra with the composition of the blends. It is worth noting the use of the SPA variable selection algorithm to reduce the number of variables in the problem and enhance the performance of the PLS algorithm. This paper reports preliminary results of the chemometric analysis applied to NR/SBR blends. Based on the results attained it is foreseen that this new approach can open an original research topic in the analysis of polymer science.

\section{The Analyzed Samples}

The blends studied are composed by NR (Malaysian standard SMR-20; isometric variant cis-1,4) and SBR (Arpol
E-SBR-1502 with $23.5 \%$ of styrene provided by Petrobras) with molecular weight of $178800 \mathrm{~g} / \mathrm{mol}$ and $128300 \mathrm{~g} / \mathrm{mol}$ and density of $0.917 \mathrm{~g} / \mathrm{cm}^{3}$ and $0.935 \mathrm{~g} / \mathrm{cm}^{3}$ for NR and SBR, respectively.

A total set of eleven samples with different NR/SBR proportions were prepared, as shown in Table 1.

All the recipes were based on the same vulcanization system, composed of $2.25 \mathrm{phr}$ (parts per hundred of rubber) of sulfur, $0.7 \mathrm{phr}$ of accelerator (N-t-butyl-2-benzothiazole sulfonamide, TBBS), 2 phr of stearic acid and 5 phr of zinc oxide, the latter two acting as activators.

The samples were prepared as follows. In a first step, the NR material, as received, was dissolved in toluene to remove the impurities by precipitation. Next, the purified solution was extracted and the solvent was evaporated at room temperature during two days to obtain a purified NR film. To prepare the compounds, the elastomer-to-solvent proportions were $18 \mathrm{~g} / \mathrm{dm}^{3}$ for NR (purified) and $20 \mathrm{~g} / \mathrm{dm}^{3}$ for SBR, respectively. To prepare each blend, both solutions of NR and SBR were mixed with the chosen proportions of each elastomer and the required chemicals, according to the recipe. The mixture was further homogenized by applying mechanical stirring and ultrasonic treatment in a bath. Finally, the solvent was evaporated at room temperature for 3 days until a constant weight was attained. The accuracy in the rubber composition was estimated in about $2 \%$.

\section{Data Acquisition By Means of DSC and FTIR Spectroscopy}

The analysis of the thermal behavior of the NR/SBR blends was made with a differential scanning calorimeter

Table 1. Samples analyzed

\begin{tabular}{|c|c|c|}
\hline Designation & Composition & Variabley \\
\hline NR & $100 \% \mathrm{NR}$ & 1.0 \\
\hline $90 \mathrm{NR} / 10 \mathrm{SBR}$ & $\begin{array}{c}90 \% \text { NR }-10 \% \\
\text { SBR }\end{array}$ & 0.9 \\
\hline $80 \mathrm{NR} / 20 \mathrm{SBR}$ & $\begin{array}{c}80 \% \text { NR }-20 \% \\
\text { SBR }\end{array}$ & 0.8 \\
\hline $70 \mathrm{NR} / 30 \mathrm{SBR}$ & $\begin{array}{c}70 \% \mathrm{NR}-30 \% \\
\text { SBR }\end{array}$ & 0.7 \\
\hline $60 \mathrm{NR} / 40 \mathrm{SBR}$ & $\begin{array}{c}60 \% \mathrm{NR}-40 \% \\
\text { SBR }\end{array}$ & 0.6 \\
\hline $50 \mathrm{NR} / 50 \mathrm{SBR}$ & $\begin{array}{c}50 \% \mathrm{NR}-50 \% \\
\mathrm{SBR}\end{array}$ & 0.5 \\
\hline $40 \mathrm{NR} / 60 \mathrm{SBR}$ & $\begin{array}{c}40 \% \mathrm{NR}-60 \% \\
\text { SBR }\end{array}$ & 0.4 \\
\hline $30 \mathrm{NR} / 70 \mathrm{SBR}$ & $\begin{array}{c}30 \% \mathrm{NR}-70 \% \\
\text { SBR }\end{array}$ & 0.3 \\
\hline $20 \mathrm{NR} / 80 \mathrm{SBR}$ & $\begin{array}{c}20 \% \mathrm{NR}-80 \% \\
\text { SBR }\end{array}$ & 0.2 \\
\hline $10 \mathrm{NR} / 90 \mathrm{SBR}$ & $\begin{array}{c}10 \% \mathrm{NR}-90 \% \\
\text { SBR }\end{array}$ & 0.1 \\
\hline SBR & $100 \% \mathrm{SBR}$ & 0.0 \\
\hline
\end{tabular}


Q20 from TA Instruments. Indium and mercury were used as reference materials to calibrate the temperature and heat flow readings of the instrument. Blend samples of about 4 mg were cut from the cured specimens. The calorimeter was programmed for a heating/cooling/heating cycle between $-89{ }^{\circ} \mathrm{C}$ and $-3{ }^{\circ} \mathrm{C}$ with a rate of $10{ }^{\circ} \mathrm{C} / \mathrm{min}$. Measurements were performed under an argon atmosphere with a constant flux of $50 \mathrm{~mL} / \mathrm{min}$. Each DSC curve was composed of 344 temperature points. The DSC data points of the 11 analyzed NR-SBR blends were concentrated in a data matrix with 11 rows (NR-SBR blends) and 344 columns (heat flux at each temperature). This numerical matrix was saved and transferred to the computer for further mathematical processing.

FTIR spectroscopy is recognized as a nondestructive instrumental technique with appealing features, including fast response without the need of any sample preparation, thus avoiding the use of chemical products or reagents, while offering an acceptable cost per analysis ${ }^{25}$. Because of the large amount of data in each FTIR spectrum (1650 data points) the analysis of such information is a challenging task, thus requiring the use of suitable multivariate data processing algorithms. FTIR (Fourier transform infrared) spectra were acquired by using a Nicolet 8700 FT-IR spectrometer from Fisher Scientific in the ATR (attenuated total reflectance) mode. The FTIR-ATR spectra of the 11 blends of NR and SBR studied in this work, were registered at $25 \pm 1^{\circ} \mathrm{C}$, over the wavenumber range $3825-525 \mathrm{~cm}^{-1}$ wavelengths. The spectrum of each sample was obtained by averaging 64 scans per sample. Each spectrum consists of 1650 wavelengths spaced by $2 \mathrm{~cm}^{-1}$. The FTIR-ATR spectral content of the 11 analyzed NR-SBR blends were concentrated in a data matrix with 11 rows (NR-SBR blends) and 1650 columns (reflectances at each of the 1650 wavelengths). This numerical matrix was saved and transferred to the computer for further mathematical processing.

\section{Multivariate Data Analysis Methods}

The successive projections algorithm (SPA) was developed by Araújo et $a l .{ }^{26}$ to deal with spectroscopic multicomponent data. It is applied as a variable selection algorithm to reduce the number of explanatory variables $x$, which often are multicollinear and thus some of them have no influence on the response variable $y$ or are highly affected by random noise. The SPA is a guided forward variable selection method since it starts with only one variable and incorporates new variables or wavelengths-temperatures until reaching a preselected number of variables within the interval $[n-2, m]$, $n$ being the total number of samples in the calibration set and $m$ the number of variables or wavelengths-temperatures in the spectra. The variables are selected based on minimizing variable collinearity or information content redundancy ${ }^{23}$. A matrix $X$ of samples of dimensions $[n, m]$ was obtained for both the DSC and FTIR data, where $n=11$ samples and $m=344$ temperatures and $m=1650$ wavelengths for DSC and FTIR registers, respectively. First- and second-derivatives of the DSC and FTIR data were calculated by applying a five-point moving average to maximize the signal-to-noise ratio during differentiation using the Savitzky-Golay algorithm. Therefore the first- and second-derivative matrixes $X 1 d$ and $X 2 d$ are composed of $[n, m-5]$ and $[n, m-10]$ components, respectively. The PLS models required to predict the composition of the samples were generated by using Matla ${ }^{\circledR}$ codes programmed by the authors of this paper. The SPA variable selection algorithm is applied previous to the application of the PLS regression method.

The PLS regression algorithm is a multivariate data analysis technique widely applied in econometrics, social sciences, chemometrics and other areas ${ }^{27}$. PLS offers better performance and robustness compared to other multivariate regression methods such as MLR and PCR, since model parameters do not alter significantly when adding or removing calibration samples from the initial model. Advantages of PLS include the ability to handle collinearity among the measured variables and robustness against noise interfering measured data. Collinearity often occurs when dealing with spectral information ${ }^{28}$. During the calibration phase, the PLS algorithm generates a linear model by projecting both the matrix $X$ comprising the measured or explanatory variables and the response variable $y$ to a new space. PLS is particularly appropriate when the matrix $X$ of predictors includes less samples or observations than variables, and when the predictors are collinear.

When dealing with a sufficient number of samples, to check the behavior and accuracy of a regression model, the entire body of samples is often split into the calibration and prediction set ${ }^{29}$. The calibration model uses the calibration set of samples to generate a predictive model. Once the model is settled, the prediction samples are used to validate the accuracy of the model. However, this paper deals with a reduced number of samples, so this strategy is no applicable. To obtain a reliable regression model based on the reduced number of samples available, the model is calibrated via cross-validation. Cross-validation does not use the entire data set to generate a regression model; instead cross-validation removes some samples from the entire data set before the calibration stage. The removed data are used as new data to validate the performance of the regression model. This paper deals with the leave-one-out cross-validation (LOOCV) method, since it is among the simplest and most commonly used versions of cross-validation in chemometrics. The LOOCV algorithm is based on the following steps,

- $\quad$ For $k=1$ to $n$ ( $n$ is the number of calibration samples)

- $\quad$ Remove the $k$-th sample from the data set.

- Generate a regression model based on the remaining $n$-1 samples.

- Test the removed sample and calculate the error. Finally, the mean error over all $n$ samples is calculated. 
LOOCV is a useful algorithm because it utilizes all the available data efficiently, without any waste of spectral-thermal information, so it is specially indicated when dealing with a reduced number of samples.

The determination of the precise number of PLS components to be retained in the model is a key factor in building a robust regression model, since it allows avoiding over-fitting and minimizing the presence of signal noise in the model. The commonly accepted criterion to determine the number of factors is that the chosen number will result in minimal prediction error ${ }^{30}$.

Different statistics can be used to determine the performance and accuracy of multivariate regression methods. In this paper the coefficient of determination $R^{2}$ and the root mean square error (RMSE) are used for this purpose, which are calculated as follows ${ }^{27}$,

$$
\begin{gathered}
R^{2}=1-\frac{\sum_{i=1}^{n}\left(y_{i}-\hat{y}_{i}\right)^{2}}{\sum_{i=1}^{n}\left(\hat{y}_{i}-\overline{\hat{y}}_{i}\right)^{2}} \\
R M S E=\sqrt{\frac{1}{n} \cdot \sum_{i=1}^{n}\left(y_{i}-\hat{y}_{i}\right)^{2}}
\end{gathered}
$$

\section{FTIR spectra}

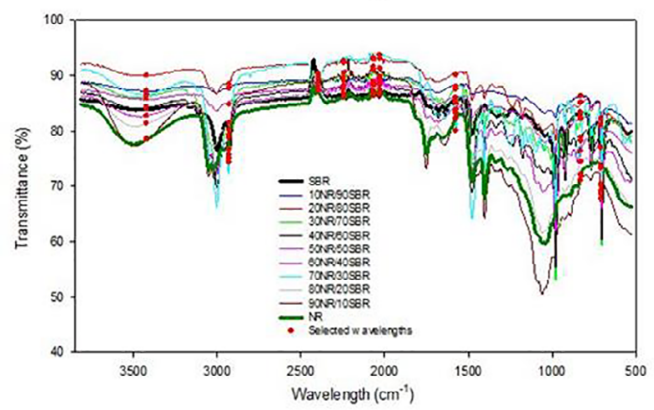

DSC thermograms

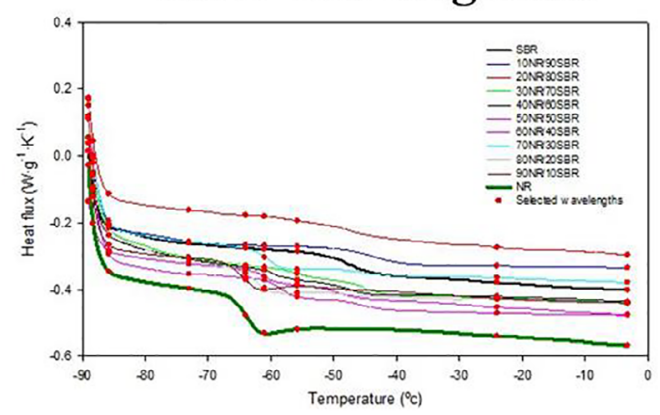

FTIR or DSC

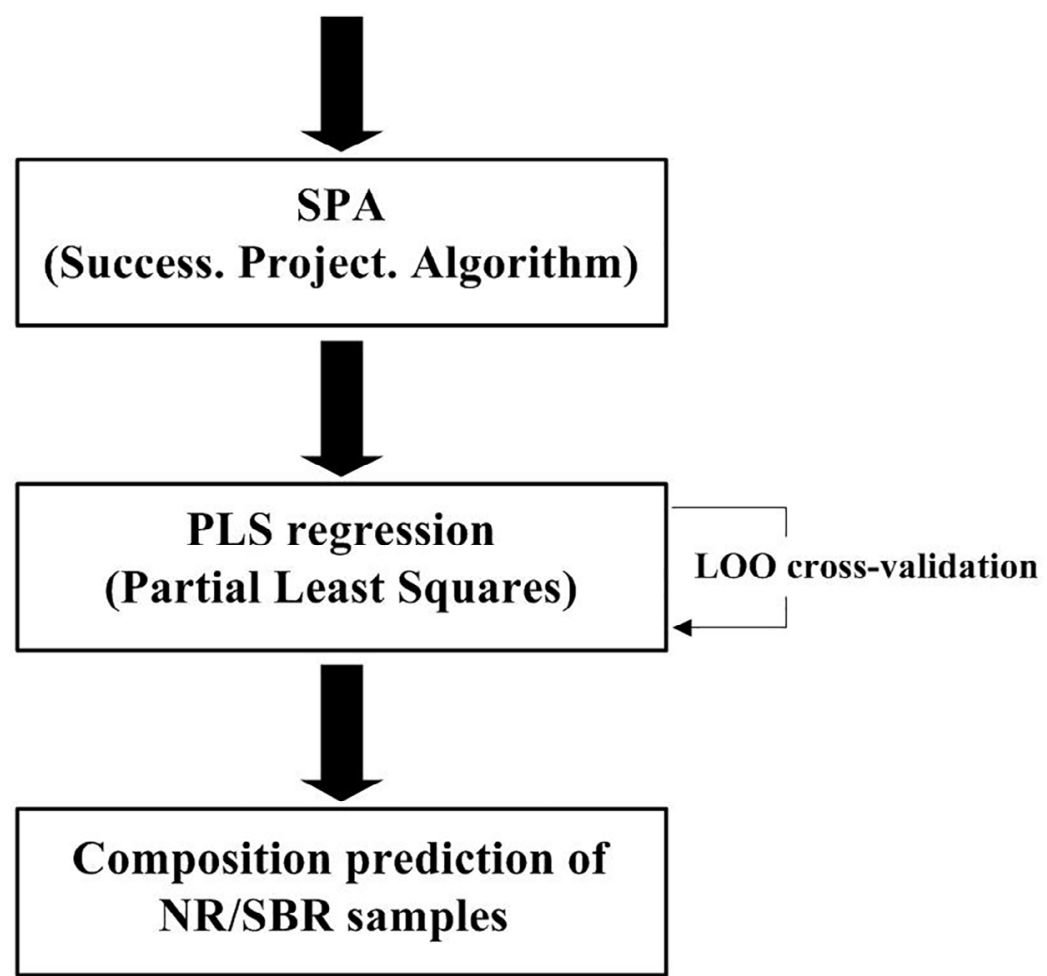

Figure 1. Flowchart summarizing the mathematical methods applied in this paper. 
being $y_{i}$ and $\hat{y}_{i}$, respectively, the reference composition of the samples studied and the composition predicted by the statistical calibration method, $n$ being the number of samples dealt with. It is noted that $\overline{\hat{y}}_{i}$ is the average value of $\hat{y}_{i}$ over the $n$ analyzed samples.

Fig. 1 summarizes the approach applied in this paper.

\section{Results and Discussion}

This section presents the results attained with both the DSC and FTIR techniques.

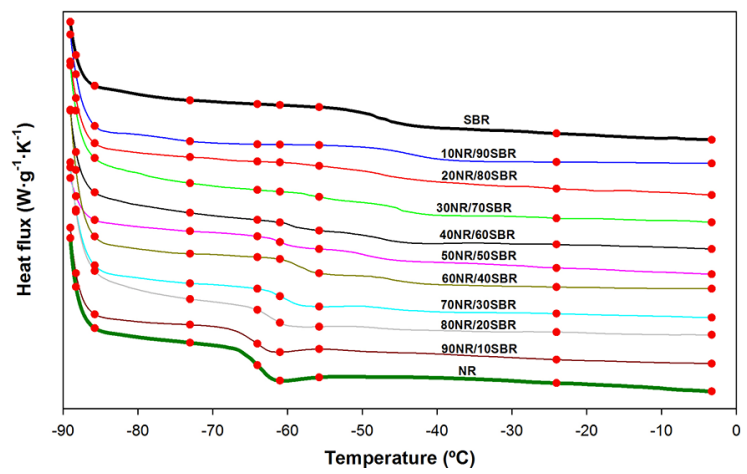

Figure 2. DSC curves expressing the heat flow against temperature $\left({ }^{\circ} \mathrm{C}\right)$ of the NR/SBR blends and $9(n=11, n-2=9)$ temperature points automatically selected by the SPA algorithm. The red dots indicate the selected temperatures.

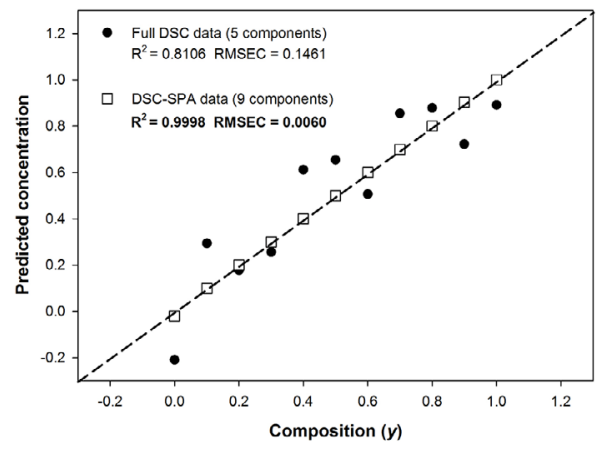

(a)
Figure 2 shows the DSC curves of the eleven analyzed samples. Although some differences among samples are appreciated, they do not allow to determine the composition of the NR/SBR blends. Thus, the application of suitable mathematical algorithms to determine the composition of such samples is required. It is noted that the DSC curves have been displaced along the vertical axis to show more clearly the small differences among the samples.

In some of the elastomeric binary samples there are cases in which the two phases are miscible, and a single glass transition temperature $T_{g}$ is observed. However, when dealing with two immiscible components, two $T_{g}$ values are usually observed, each one for each of the two components. A shift in the glass transition temperature corresponding to an elastomer in a mixture with respect to its pure state, indicates partial solubility with the other component ${ }^{31}$. Fig. 2 shows the glass transition temperature $T_{g}$ of all vulcanized compounds, either pure elastomers or mixtures. It is observed that, for most blends, it is possible to distinguish the values of $T_{g}$ of each phase. This is because the two analyzed rubber compounds are immiscible.

Figures 3 show the correlations between the NR/ SBR composition predicted by PLS algorithm (from the information of the DSC curves and their derivatives with and without application of the SPA algorithm) and the measured composition.

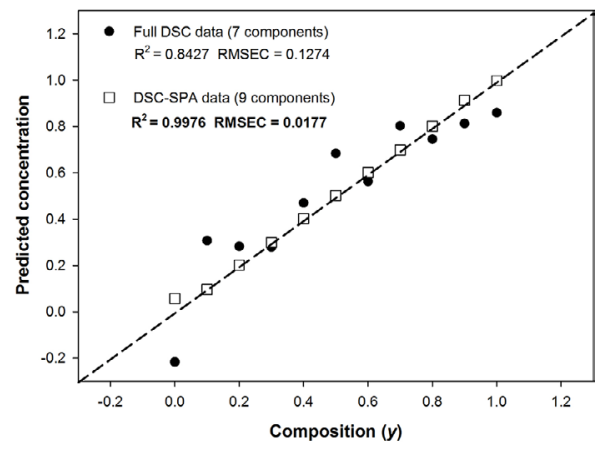

(b)

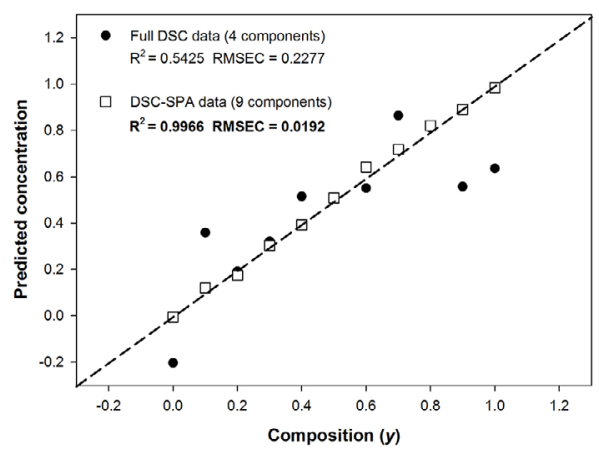

(c)

Figure 3. DSC results. Generalized correlation for the calibration samples after applying the PLS with meancentered preprocessing and full LOOCV. a) No derivative mode. b) First-derivative mode. c) Second-derivative mode. 
Results from Figure 3 clearly indicate the great performance improvement when applying the SPA variable selection algorithm previous to the PLS regression method. As seen in the results summarized in Figure 3, due to the great reduction of the number of temperatures, more PLS components must be retained when applying the SPA algorithm to obtain an accurate result. Comparing the results presented in Figure 3, although all treatments (no derivative, first derivative and second derivative modes) provide good results, the better results are attained when dealing with the DSC data in the no derivative mode.

Figure 4 shows the FTIR spectra of the eleven analyzed samples with the characteristic spectral bands. Similarly as done in Figure 2, the FTIR spectra have been displaced along the vertical axis to show more clearly the small differences among the samples. Once again, although some differences can be seen among samples, a direct deduction of the blends composition is not possible. Thus, it is necessary to apply appropriate multivariate mathematical algorithms to determine the composition of such samples.

From the FTIR reflectance spectra it is possible to identify the spectral bands associated to the pure NR vulcanized sample. The $1375 \mathrm{~cm}^{-1}$ band is related to the symmetrical deformation of the $\mathrm{CH}_{3}$ groups. The $1446 \mathrm{~cm}^{-1}$ spectral band has two main contributions, the symmetrical deformation of the scissor $\mathrm{CH}_{2}$ groups and the asymmetrical deformation of the $\mathrm{CH}_{3}$ groups. The peak corresponding to $2869 \mathrm{~cm}^{-1}$ is due to the symmetrical stretching of the $\mathrm{CH}_{2}$ and $\mathrm{CH}_{3}$ groups, whereas at $2931 \mathrm{~cm}^{-1}$ there is an asymmetrical stretching of the $\mathrm{CH}_{2}$ groups. Finally, the peak corresponding at 2962 $\mathrm{cm}^{-1}$ is attributed to the asymmetrical stretching of the $\mathrm{CH}_{3}$ groups ${ }^{5,32}$. Other spectral bands are associated to the sulfur oxidation products and other oxidation products.

The $700 \mathrm{~cm}^{-1}$ and $758 \mathrm{~cm}^{-1}$ spectral bands of the vulcanized SBR sample, are attributed to the out-of-plane deformation of the $\mathrm{C}=\mathrm{C}$ and $=\mathrm{C}-\mathrm{H}$ groups of the benzene ring, respectively. The $964 \mathrm{~cm}^{-1}$ and $993 \mathrm{~cm}^{-1}$ spectral bands

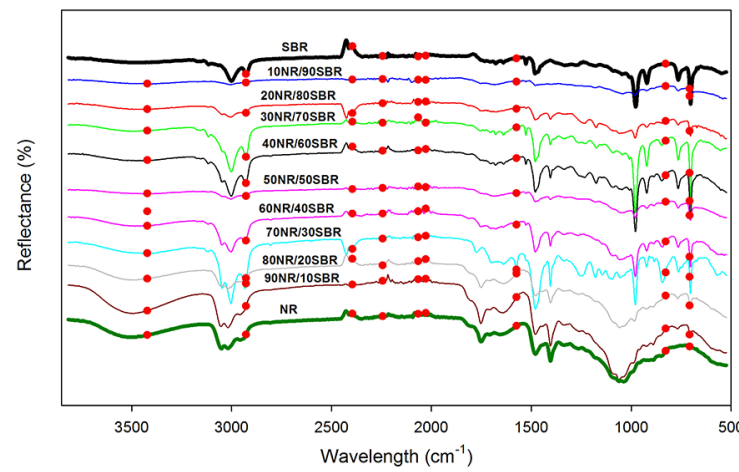

Figure 4. FTIR reflectance spectra of the analyzed NR/SBR blends. Full spectral data and $9(n=11, n-2=9)$ wavelengths automatically selected by the SPA algorithm. The red dots indicate the selected wavelengths. are due to the out-of-plane deformation of the $\mathrm{CH}$ and $=\mathrm{C}-\mathrm{H}$ groups of the trans-1,4-butadiene. The $1493 \mathrm{~cm}^{-1}, 1600 \mathrm{~cm}^{-1}$ and $3026 \mathrm{~cm}^{-1}$ spectral bands are related to the stretching of the $\mathrm{CH}$ group of the benzene ring. The $3070 \mathrm{~cm}^{-1}$ spectral band is attributed to the asymmetrical stretching of the $\mathrm{CH}_{2}$ group of the 1,2-butadiene ${ }^{5,6,32}$.

Figure 4 also shows the spectra corresponding to the elastomeric blends. They show that both the shape and intensity of the spectral bands change according to the composition of the blend, thus being dominant the elastomer with higher proportion.

Figures 5 show the NR/SBR composition predicted by the PLS algorithm from the information of the FTIR spectra and their derivatives, with and without application of the SPA variable selection algorithm (white squares and black circles, respectively).

Results from Figure 5 show the notable improvement of the accuracy when applying the SPA algorithm previous to the PLS regression method. Similarly as occurred with the DSC curves data, the SPA algorithm requires retaining more PLS components. The results summarized in Figure 5, show that all treatments (no derivative, first derivative and second derivative modes) provide good and similar results.

\section{Conclusions}

In this study, different blends of vulcanized NR and BR with variable blend ratios have been analyzed. All blend samples were analyzed by means of the DSC and FTIR instrumental techniques. Both DSC and FTIR are fast, do not require a previous treatment of the samples and are environmentallyfriendly instrumental techniques. Afterwards, suitable multivariate chemometric methods (SPA variable selection algorithm, PLS regression and LOO cross-validation) were applied to generate a mathematical correlation between the registers and the composition of the blends.

This new approach, consisting in applying the SPA algorithm previous to generate the PLS regression model, has allowed to obtain good results for both the FTIR technique, which has already been widely applied in the identification and quantitative analysis of samples, and for the DSC instrumental method which is widely applied in the rubber industry. By applying both techniques it has been possible to determine the composition of the analyzed rubber blends with accuracy.

This work has shown that DSC curves of rubber blends provides a large amount of information, which treated properly, can not only qualify the analyzed samples, but also their quantification. Results attained encourage us to continue this research in order to extrapolate the method applied to various blends of elastomers of industrial interest. 


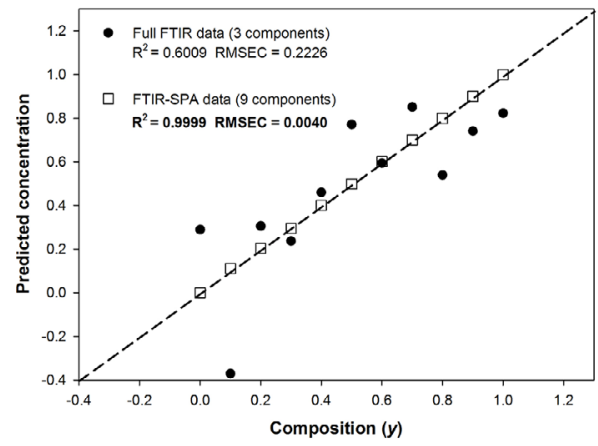

(a)

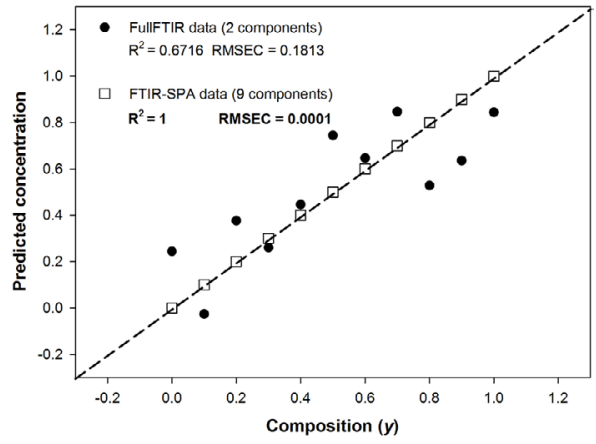

(b)

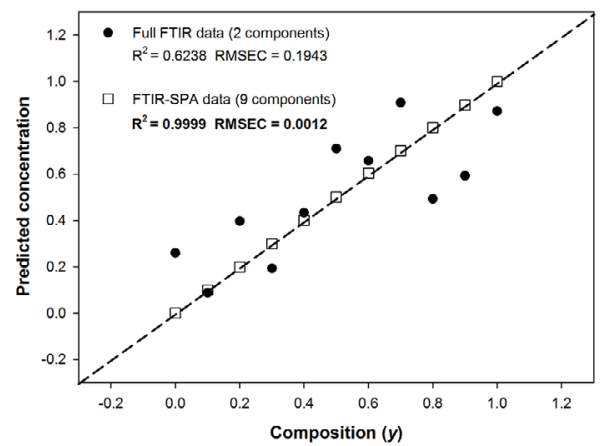

(c)

Figure 5. FTIR results. Generalized correlation for the calibration samples after applying the PLS with mean-centered preprocessing and full LOOCV. a) No derivative mode. b) First-derivative mode. c) Secondderivative mode.

\section{7 . Acknowledgment}

This work was partially supported by UBACyT 20020120100051 (Argentina).

\section{References}

1. van Beilen JB, PoirierY. Establishment of new crops for the production of natural rubber. Trends in Biotechnology. 2007;25(11):522-529.

2. Lee HG, Kim HS, Cho ST, Jung IT, Cho CT. Characterization of Solution Styrene Butadiene Rubber (SBR) Through the Evaluation of Static and Dynamic Mechanical Properties and Fatigue in Silica-Filled Compound. Asian Journal of Chemistry [Internet]. 2013 [cited 2017 Mar 23];25(9):5251-5256. Available from: http://www.asianjournalofchemistry.co.in/User/ViewFreeArticle. aspx?ArticleID=25_9_128

3. González N, del Àngels Custal M, Lalaouna S, Riba JR, Armelin E. Improvement of dielectric properties of natural rubber by adding perovskite nanoparticles. European Polymer Journal [Internet]. 2016 Feb 1 [cited 2016 Jun 16];75:210-222. Available from: http://www. sciencedirect.com/science/article/pii/S0014305715301026

4. Mangaraj D. Elastomer Blends. Rubber Chemistry and Technology [Internet]. 2002 Jul [cited 2016 Nov 2];75(3):365-427. Available from: http://rubberchemtechnol.org/doi/abs/10.5254/1.3547677

5. Lee YS, Lee WK, Cho SG, Kim I, Ha CS. Quantitative analysis of unknown compositions in ternary polymer blends: A model study on NR/SBR/BR system. Journal of Analytical and Applied Pyrolysis [Internet]. 2007 [cited 2017 Apr 7];78(1):85-94. Available from: http:// www.sciencedirect.com/science/article/pii/S0165237006000623
6. Fernández-Berridi MJ, González N, Mugica A, Bernicot C. PyrolysisFTIR and TGA techniques as tools in the characterization of blends of natural rubber and SBR. Thermochimica Acta [Internet]. 2006 [cited 2017 Apr 10];444(1):65-70. Available from: http://www. sciencedirect.com/science/article/pii/S0040603106001110

7. Puentes J, Restrepo-Zapata NC, Chaloupka A, Duddleston LJL, Rudolph N, Osswald TA. Quasi-isothermal DSC testing of epoxy adhesives using initial fast heating rates. Journal of Applied Polymer Science [Internet]. 2017 Nov 5 [cited 2017 Dec 10];134(42):45425. Available from: http://doi.wiley.com/10.1002/app.45425

8. Hourston DJ, Song M. Quantitative characterization of interfaces in rubber-rubber blends by means of modulated-temperature DSC. Journal of Applied Polymer Science [Internet]. 2000 Jun 20 [cited 2017 Apr 7];76(12):1791-1798. Available from: http://doi.wiley. com $/ 10.1002 / \% 28$ SICI\%291097-4628\%2820000620\%2976\%3A1 2\%3C1791\%3A\%3AAID-APP9\%3E3.0.CO\%3B2-J

9. EAG Laboratories. Characterization of Polymers using Differential Scanning Calorimetry (DSC). San Diego: EAG Laboratories; 2016. p. 1-5.

10. Danon B, Görgens J. Determining rubber composition of waste tyres using devolatilisation kinetics. Thermochimica Acta [Internet]. 2015 [cited 2016 Jul 16];621:56-60. Available from: https://www. sciencedirect.com/science/article/abs/pii/S0040603115004141

11. Razavizadeh M, Jamshidi M. Effects of methylene diphenyl diisocyanate on the physical, mechanical, and vulcanization properties of nitrile rubber. Journal of Applied Polymer Science [Internet]. $2017 \mathrm{Sep}$ 5 [cited 2018 Apr 19];134(33):45200. Available from: http://doi. wiley.com/10.1002/app.45200 
12. Ortiz Vélez A, Siles Alvarado S, Avendaño-Gómez JR. Cure behavior and kinetic study of diglycidyl ether of bisphenol A with a tertiary amine salt by differential scanning calorimetry. Polymer Engineering and Science [Internet]. 2018 May [cited 2018 Apr 19]; 58(5):784-792. Available from: http://doi.wiley. com/10.1002/pen.24614

13. Wesolowski M, Rojek B. Thermogravimetric detection of incompatibilities between atenolol and excipients using multivariate techniques. Journal of Thermal Analysis and Calorimetry [Internet]. 2013 Mar 19 [cited 2016 Mar 3];113(1):169-177. Available from: https://link.springer.com/ article/10.1007/s10973-013-3070-y

14. Run M, Yao C, Wang Y. Morphology, isothermal and non-isothermal crystallization kinetics of poly(methylene terephthalate). European Polymer Journal [Internet]. 2006 [cited 2017 Apr 8];42(3):655-662. Available from: http://www. sciencedirect.com/science/article/pii/S0014305705003356

15. Riaz U, Ashraf SM. Characterization of Polymer Blends with FTIR Spectroscopy. In: Thomas S, Grohens Y, Jyotishkumar P, eds. Characterization of Polymer Blends [Internet]. Weinheim: Wiley-VCH; 2014 [cited 2017 Apr 8]. p. 625-678. Available from: http://doi.wiley.com/10.1002/9783527645602.ch20

16. Šturcová A, Kratochvíl J, Dybal J, Sikora A. Fourier-transform infrared spectroscopic study of a fractional-complexed polymer blend. European Polymer Journal [Internet]. 2014 [cited 2017 Apr 8];59:200-207. Available from: http://www.sciencedirect. com/science/article/pii/S0014305714002602

17. Choi SS, Kwon HM, Kim Y, Ko E, Kim E. Determination of bound rubber composition of filled SBR/BR blend compounds by analysis of the unbound rubber composition and bound rubber content. Polymer Testing [Internet]. 2017 [cited 2017 Apr 8];59:414-422. Available from: http://www.sciencedirect. com/science/article/pii/S0142941817300879

18. Dolar D, Drašinac N, Košutić K, Škorić I, Ašperger D. Adsorption of hydrophilic and hydrophobic pharmaceuticals on $\mathrm{RO} / \mathrm{NF}$ membranes: Identification of interactions using FTIR. Journal of Applied Polymer Science [Internet]. 2017 Feb 5 [cited 2017 Dec 10];134(5):44426. Available from: http://doi.wiley.com/10.1002/app.44426

19. Fang W, Zeng X, Lai X, Li H, Chen W, Zhang Y. Thermal degradation mechanism of addition-cure liquid silicone rubber with urea-containing silane. Thermochimica Acta [Internet]. 2015 [cited 2017 Apr 10];605:28-36. Available from: https://www.sciencedirect.com/science/article/abs/pii/ S004060311500060X

20. Mansilla MA, Valentín JL, López-Manchado MA, GonzálezJiménez A, Marzocca AJ. Effect of entanglements in the microstructure of cured NR/SBR blends prepared by solution and mixing in a two-roll mill. European Polymer Journal. 2016;81:365-375.

21. Mansilla MA, Marzocca AJ, Macchi C, Somoza A. Influence of vulcanization temperature on the cure kinetics and on the microstructural properties in natural rubber/styrene-butadiene rubber blends prepared by solution mixing. European Polymer Journal. 2015;69:50-61.
22. Mansilla MA. Influence of the microstructure on the mechanical an thermal properties of Natural Rubber and Styrene Butadiene Rubber. [Thesis]. Buenos Aires: Facultad de Ciencias Exactas y Naturales, Universidad de Buenos Aires; 2012 [Internet] [cited 2015 Nov 6]. Available from: http://digital.bl.fcen.uba.ar/gsdl282/cgi-bin/library.cgi? $\mathrm{a}=\mathrm{d} \& \mathrm{c}=$ tesis\&d=Tesis_5117_Mansilla

23. Galvão RKR, Pimentel MF, Araujo MCU, Yoneyama T, Visani $\mathrm{V}$. Aspects of the successive projections algorithm for variable selection in multivariate calibration applied to plasma emission spectrometry. Analytica Chimica Acta. 2001;443(1):107-115.

24. Khanmohammadi M, Soleimani M, Morovvat F, Garmarudi AB, Khalafbeigi M, Ghasemi K. Simultaneous determination of paracetamol and codeine phosphate in tablets by TGA and chemometrics. Thermochimica Acta [Internet]. $2012 \mathrm{Feb}$ [cited 2016 Apr 21];530:128-132. Available from: http://www. sciencedirect.com/science/article/pii/S0040603111005983

25. Riba JR, Canals T, Cantero R. Recovered Paperboard Samples Identification by Means of Mid-Infrared Sensors. IEEE Sensors Journal [Internet]. 2013 Jul 1 [cited 2015 Nov 8];13(7):27632770. Available from: http://ieeexplore.iee.org/articleDetails. jsp?arnumber $=6497472$

26. Araújo MCU, Saldanha TCB, Galvão RKH, Yoneyama T, Chame $\mathrm{HC}$, Visani V. The successive projections algorithm for variable selection in spectroscopic multicomponent analysis. Chemometrics and Intelligent Laboratory Systems. 2001;57(2):65-73.

27. Riba JR, Canals T, Cantero R. Determination of the recoveredfiber content in paperboard samples by applying mid-infrared spectroscopy. Applied Spectroscopy [Internet]. 2015 Apr [cited 2015 Nov 8];69(4):442-450. Available from: http://www.ncbi. nlm.nih.gov/pubmed/25742130

28. Zhou Y, Chen Z, Liu T, Mao J. Extended Canonical Variates Analysis for Wine Origin Discrimination by Using Infrared Spectroscopy. Chemistry Letters [Internet]. 2016 May 5 [cited 2016 Nov 5];45(5):564-566. Available from: http://www.journal. csj.jp/doi/10.1246/cl.160135

29. Riba Ruiz JR, Canals Parelló T, Cantero Gómez R. Identification of NR and EPDM Samples by Means of Thermogravimetric Analysis and Multivariate Methods. IEEE Sensors Journal [Internet]. 2016 Nov [cited 2016 Nov 3];16(21):7705-7712. Available from: http://ieeexplore.ieee.org/document/7552559/

30. Lorber A, Kowalski BR. Alternatives to Cross-Validatory Estimation of the Number of Factors in Multivariate Calibration. Applied Spectroscopy [Internet]. 1990 Nov 1 [cited 2016 Nov 4];44(9):1464-1470. Available from: http://asp.sagepub.com/ lookup/doi/10.1366/0003702904417788

31. Turi EA. Thermal Characterization of Polymeric Materials [Internet]. San Diego: Academic Press; 1981 [cited 2017 Apr 8]. 972 p. Available from: http://www.sciencedirect.com/science/ book/9780127037806

32. Gunasekaran S, Natarajan RKK, Kala A. FTIR spectra and mechanical strength analysis of some selected rubber derivatives. Spectrochimica Acta Part A: Molecular and Biomolecular Spectroscopy [Internet]. 2007 Oct [cited 2016 Jan 27];68(2):323330. Available from: http://www.sciencedirect.com/science/ article/pii/S1386142506007074 